\section{PROCESOS FÍSICOS EN BIOTECNOLOGÍA: ENSEÑANZA Y DISEÑO}

\author{
Sergio Collado \\ Universidad de Oviedo \\ colladosergio@uniovi.es \\ Mario Díaz \\ Universidad de Oviedo \\ mariodiaz@uniovi.es
}

\section{PHYSICAL PROCESSES IN BIOTECHNOLOGY: EDUCATION AND DESIGN}

Cómo citar este artículo/Citation: Collado, S. y Díaz, M. (2014). "Procesos físicos en biotecnología: enseñanza y diseño". Arbor, 190 (768): a158. doi: http://dx.doi.org/10.3989/ arbor.2014.768n4012

Recibido: 9 junio 2014. Aceptado: 14 julio 2014.

RESUMEN: Los procesos de base física son fundamentales para las aplicaciones de la Biotecnología, en etapas como, por ejemplo, la preparación de los sustratos, la separación y purificación de los bioproductos o la transferencia de energía hacia o desde los biorreactores. Se discuten aquí los factores más importantes en el diseño de operaciones de base física en un proceso de tipo biotecnológico, se tratan las especificidades al trabajar con moléculas de base biológica, y se analiza cómo se enseña el diseño de operaciones básicas en los grados en Biotecnología de distintas universidades españolas.

PALABRAS CLAVE: Biotecnología; dinámica de fluidos; diseño; enseñanza; fenómenos de transporte; ingeniería de bioprocesos; operación unitaria; proceso físico; transferencia de materia; transmisión de calor.
Copyright: (c) 2014 CSIC. Este es un artículo de acceso abierto distribuido bajo los términos de la licencia Creative Commons Attribution-Non Commercial (by-nc) Spain 3.0.
ABSTRACT: Physically-based processes are critical for Biotechnology applications, in stages such as, for example, the preparation of the substrates, the separation and purification of bioproducts or the energy transfer to or from the bioreactors. The most significant factors during the design of operations with physical base are discussed here, the specifics when working with bio-based molecules are dealt and how the design of the unit operations is to be taught in the Bachelor on Biotechnology at various Spanish universities is also analysed.

KEYWORDS: Biotechnology; fluid dynamics; design; education; transport phenomena; bioprocesses engineering; unit operation; physical process; mass transfer; heat transfer. 


\section{INTRODUCCIÓN. PROCESOS BIOTECNOLÓGICOS}

Según los últimos datos disponibles (año 2012), el sector biotecnológico supuso para España un 7.15\% del PIB, empleó a 202.250 trabajadores y alcanzó una cifra de negocios de 76.069 millones de euros, el 26,5\% más respecto al año anterior. En los diez últimos años, el número de empresas de base biotecnológica ha aumentado un $359 \%$, con un incremento de la facturación de más del $600 \%$, manteniendo tasas de crecimiento de dos cifras a pesar de la dureza de la crisis y situándose como un sector estratégico de la economía española.

Todo lo anterior hace evidente la necesidad de continuar formando profesionales capacitados para trabajar en el sector biotecnológico. Para ello, hay que dotar a estos de una formación multidisciplinar equilibrada, la cual combine conocimientos seleccionados de química, biología, bioquímica e ingeniería química, entre otras disciplinas, permitiéndoles adquirir competencias que no se adquieren en su conjunto en ninguno de los estudios tradicionales.

La aplicación industrial, práctica, de los procesos biotecnológicos requiere de una serie de conocimientos específicos. Estos conocimientos tecnológicos conforman una ingeniería, habitualmente denominada Ingeniería de Bioprocesos, aunque en algunos lugares se la ha denominado Ingeniería Biotecnológica. La Ingeniería de Bioprocesos, como una de las herramientas de la Biotecnología, tiene por objeto el diseño y desarrollo de equipos y procesos para la fabricación de productos con valor de mercado a partir de materiales biológicos. Entre sus fines más reconocidos están: a) El diseño, desarrollo, selección, adaptación y control de nuevos bioprocesos industriales y la optimización de su funcionamiento. b)El diseño y desarrollo de nuevos biocatalizadores. c) El diseño de reactores biológicos y de operaciones básicas de extracción- purificación de producto. d) El diseño y selección de equipos que permitan la operación en condiciones óptimas en los procesos a partir de los materiales de origen biológico. e) El cambio de escala de equipos y bioprocesos.

Cuando se trata de enumerar en forma detallada los conocimientos necesarios, organizados en torno a la denominación de Ingeniería de Bioprocesos, la lista resulta muy amplia. En la Figura 1 se recoge una recopilación de conocimientos organizados en bases a la estructura de Ingeniería de Procesos, desde los aspectos microscópicos hasta los de gestión.

Figura 1. Contenidos de la Ingeniería de Bioprocesos

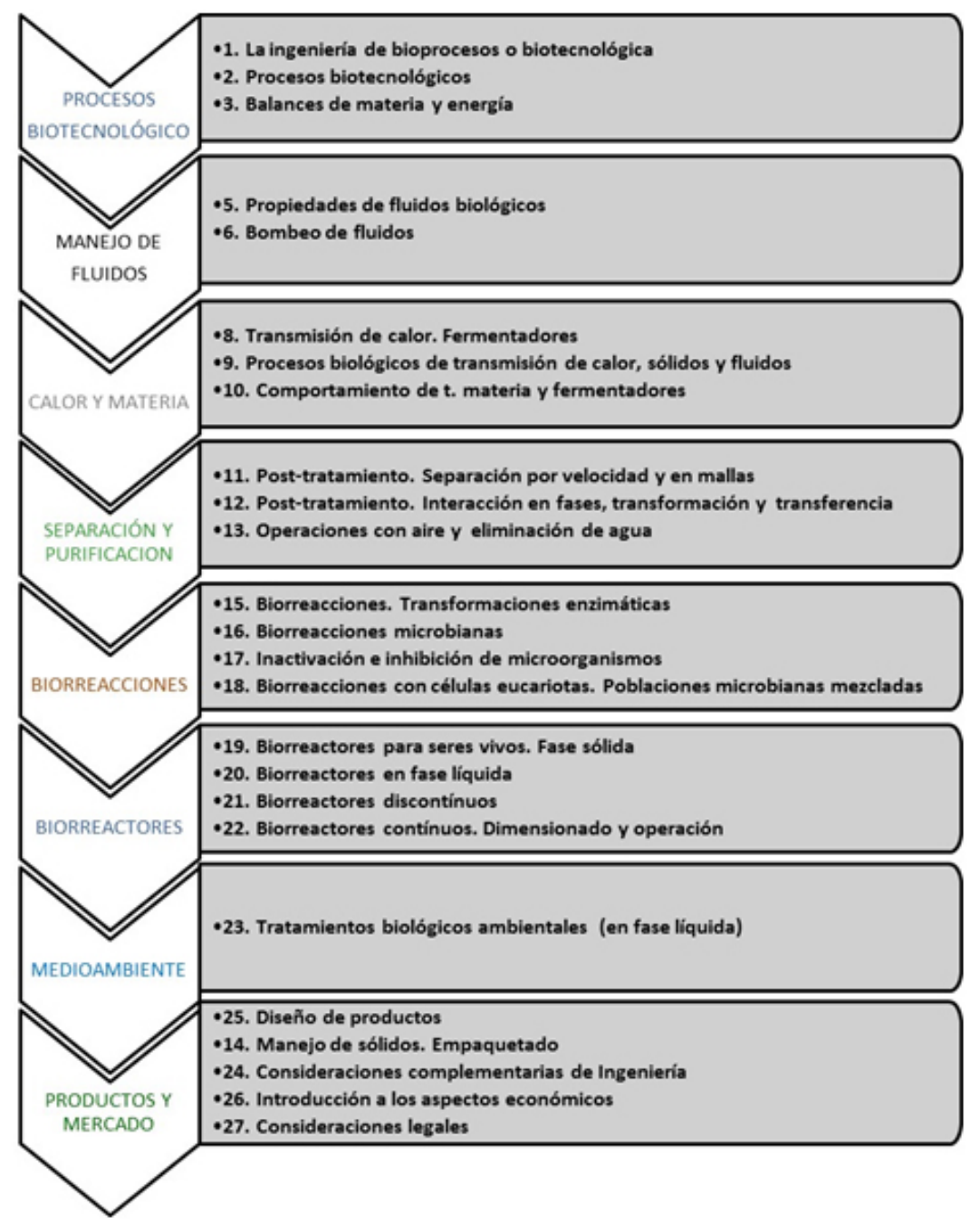




\section{PROCESOS FÍSICOS EN BIOTECNOLOGÍA. PECU- LIARIDADES}

El número de procesos u operaciones físicas utilizadas en Biotecnología es muy elevado, superior incluso al que se corresponde con procesos puramente de biotransformación. Baste pensar en todas las situaciones en las que se hace necesario mezclar, airear, separar, calentar... Estos procesos físicos suelen denominarse también operaciones básicas (o unitarias) y conforman la base de las operaciones que se realizan en biotecnología. Su conocimiento resulta imprescindible al pasar a escala industrial, aunque a escala de laboratorio en ocasiones no se requieran en exceso, bastando con el conocimiento técnico de la forma de operación.

Así pues, aunque los procesos biotecnológicos son muy diversos, presentan en común que están formados por una serie de etapas físicas básicas. Las posibles etapas u operaciones son muchas, aunque en un proceso concreto solamente se hace uso de un número pequeño de ellas. Si bien estas operaciones pueden ser específicas del bioproceso considerado, normalmente son comunes y análogas para varios procesos, lo que simplifica considerablemente el diseño.

En Biotecnología, las operaciones básicas se utilizan ampliamente al llevar a cabo el diseño de bio- procesos, tanto en las etapas previas de preparación de los sustratos, como en los ajustes térmicos, en los biorreactores, o en la separación y purificación de los bioproductos. En la Figura 2 se muestran algunos ejemplos de operaciones básicas (físicas) aplicadas a un bioproceso que incluye una etapa de biorreacción.

Los aspectos estrictamente de biorreacción suelen determinarse previamente a escala de laboratorio y de forma específica para los productos de que se trate. Las operaciones físicas suelen tener características más generales y comunes para distintos productos, aunque haya que definir bien algunas propiedades o su valoración tecnológica y económica. Entre estas propiedades se encuentran las propiedades de transporte, en particular la reología, pero también las condiciones límite de operación para evitar la transformación o deterioro de los materiales biológicos y otros aspectos de calidad y de seguridad.

La enumeración de las operaciones básicas que se aplican actualmente y su clasificación depende de los criterios de definición y de los desarrollos tecnológicos en relación a su incorporación a la industria, considerando los costes asumibles en un bioproceso. En general, la operación básica tiene como objetivo modificar las condiciones de una determinada cantidad de materia en forma más útil a nuestros fines.

Figura 2. Ejemplos de algunas operaciones básicas en un bioproceso

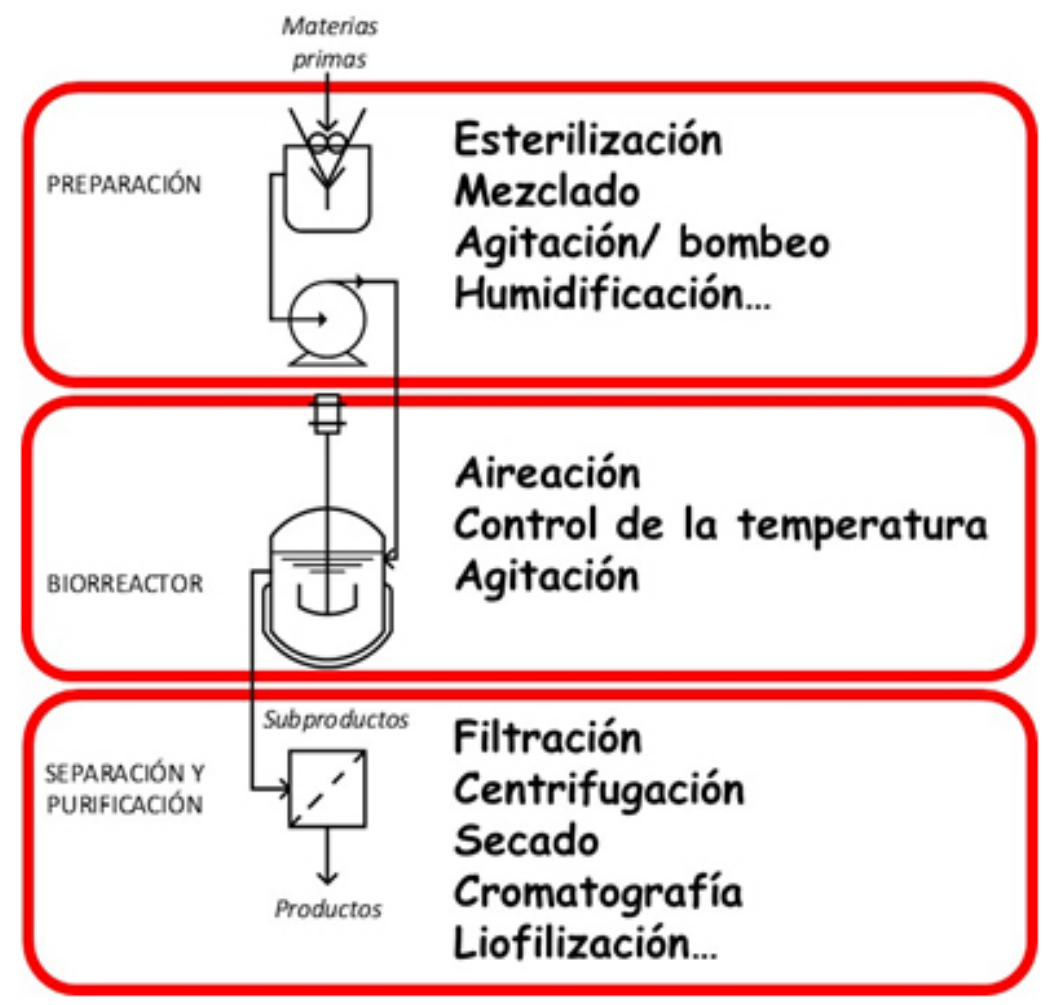


Este cambio puede hacerse principalmente por tres caminos:

- Modificando su masa o composición.

- Modificando el nivel o calidad de la energía que posee.

- Modificando sus condiciones de movimiento.

Así, las operaciones básicas se clasifican de acuerdo con la propiedad (materia, energía, cantidad de movimiento) que se transfiera en la operación y sea la más relevante en la misma. Hay también operaciones básicas en las que se transfieren simultáneamente dos o más propiedades, por ejemplo energía y materia, estando controladas estas operaciones por dos gradientes diferentes.

La división del proceso en operaciones unitarias es práctica común durante su diseño y desarrollo en el sector productivo. Esta organización ha estado menos difundida en el sector biotecnológico, probablemente por su estructuración más reciente como tal, aunque haya procesos biotecnológicos más antiguos que muchos de los procesos químicos. El sector biotecnológico, en su definición amplia, abarca otros sectores tradicionales que también han ido incorporando la notación de operaciones básicas de forma reciente.

Aunque las operaciones básicas presentan muchas características generales, uno de los errores más frecuentes a la hora de diseñar un proceso biotecnológico es aplicar directamente los conocimientos adquiri- dos en otros campos. Las características particulares de los procesos biotecnológicos hacen que determinadas operaciones básicas, tradicionalmente aplicadas en el campo químico, precisen de condiciones de operación muy diferentes o que incluso no sean aplicables al introducirlas en el campo de la Biotecnología. La Tabla I muestra algunas de estas particularidades de los procesos biotecnológicos. Específicamente, el uso de materiales biológicos, con unas propiedades muy diferentes de las materias primas no renovables o tradicionales, es uno de los factores más importantes a la hora de adaptar los conocimientos clásicos sobre operaciones básicas.

Las características específicas que tienen las operaciones físicas en Biotecnología requieren de una formación específica dirigida a los estudiantes que deban aprender a desarrollar procesos biotecnológicos. Hay bastante diversidad de estudios con este objetivo, pudiendo acercarse personas desde titulaciones como las ingenierías o los campos científicos tradicionales, u otros más alejados, como el farmacéutico o veterinario, que consideran el sector biotecnológico como un sector industrial de futuro creciente. En este trabajo haremos hincapié en la formación en procesos físicos desde la titulación en Biotecnología.

Así, a continuación trataremos, por un lado, de analizar cómo se enseña el diseño de procesos físicos en los grados en Biotecnología de distintas universidades españolas y, por otro, de discutir los factores más importantes a tener en cuenta durante el diseño

Tabla I. Alguna de las características de los procesos biotecnológicos que van a marcar el empleo de operaciones básicas durante el diseño del bioprocesos.

\begin{tabular}{|l|}
\hline \multicolumn{1}{|c|}{$\begin{array}{c}\text { Medios } \\
\text { biológicos }\end{array}$} \\
\hline - Fase líquida acuosa \\
- Empleo de \\
condiciones suaves \\
- No variación de \\
volumen \\
- Tiempos de mezcla \\
importantes \\
\\
\end{tabular}

\begin{tabular}{|l|}
\hline Sostenibilidad \\
\hline - Bajo consumo \\
energético \\
- Subproductos de \\
baja toxicidad \\
- Gran variedad de \\
operaciones \\
aplicables \\
\\
\hline
\end{tabular}

\begin{tabular}{|l|}
\hline \multicolumn{1}{|c|}{ Materias } \\
renovables \\
\hline - Diversidad de \\
sustratos \\
- Procesos fácilmente \\
modificables \\
- Oportunidad para la \\
innovación \\
- Oportunidad para la \\
diversificación \\
\\
\hline
\end{tabular}

\begin{tabular}{|l|}
\hline $\begin{array}{c}\text { Bioproductos de } \\
\text { alto valor añadido }\end{array}$ \\
\hline - Enfoque hacia \\
Ingenierla de \\
producto en lugar de \\
commodity. \\
- Traslado de \\
principios de otros \\
campos \\
- Conocimientos \\
aplicables en \\
multitud de campos \\
Operaciones poco \\
agresivas pero muy \\
específicas \\
\hline
\end{tabular}


de operaciones unitarias en un proceso de base biotecnológica, con el fin de adaptar los conocimientos tradicionales sobre diseño de procesos físicos, adquiridos principalmente en el sector químico, al proceso biológico. Para ello, se discutirá el efecto de estos factores sobre el enfoque clásico (en particular químico) de cada uno de los tipos de operaciones básicas: de transporte de cantidad de movimiento, de transmisión de calor o de transferencia de materia.

\section{ENSEÑANZA DE PROCESOS FÍSICOS EN BIOTECNOLOGÍA}

Como se ha indicado, aunque aquí trataremos la enseñanza de operaciones básicas para Biotecnología, la necesidad de esta formación se hace evidente también para cualquier titulación relacionada con la ingeniería de procesado de y con materiales biológicos, tales como Bioquímica, Tecnología de Alimentos, Ciencias Ambientales o Ingeniería Agronómica.

La Tabla II muestra las asignaturas de planes docentes de los grados de Biotecnología para distintas universidades españolas en cuyos contenidos figura el estudio de diseño de procesos físicos (operaciones unitarias). Como se puede observar en la Tabla, la carga docente relacionada con esas asignaturas para cada universidad varía de forma clara; desde grados sin carga, como el de la Universidad CEU San Pablo de Madrid hasta universidades con cargas superiores al $10 \%$ del total (> 24 ECTS), como es el caso de las de Cádiz y el País Vasco. En la mayoría de los grados, la enseñanza básica del diseño de procesos físicos se encuadra dentro de la asignatura Fundamentos (o $\mathrm{Ba}$ ses) de la Ingeniería Bioquímica (o de Bioprocesos) o análogas, la cual se suele impartir durante el segundo año del grado. La asignatura, normalmente de 6 ECTS de duración, suelen dividirse a su vez en tres bloques didácticos:

1. Balances de materia y energía.

2. Procesos de transporte.

3. Operaciones de separación/ purificación.

En varias universidades, el Bloque 3 puede suponer por sí solo una asignatura distinta, Procesos (u Operaciones) de Separación (y Purificación); este sería el caso de Cádiz, León, Sevilla, País Vasco, Rovira i Virgili de Tarragona y Autónoma de Barcelona. Lo que ya no suele ser tan frecuente es que el Bloque 2 (Procesos de transporte) sea ampliado en una asignatura posterior. Ejemplos de esto serían las asignaturas Fluidos y Transferencia de Energía (Universidad de León), Operaciones de Flujo de Fluidos y Transmisión de Calor (Universidad de Cádiz), Operaciones Básicas (Universidad Pablo de Olavide, Sevilla) o Transferencia de Materia (Universidad del País Vasco).
La asignación de la docencia de estas asignaturas, corresponde en la mayoría de los casos al área de Ingeniería Química. De hecho, la asignatura Fundamentos (o Bases) de la Ingeniería Bioquímica (o de Bioprocesos) no se imparte en las universidades de Barcelona, Vic y Zaragoza, pero sus contenidos son incluidos en el temario de la asignatura Ingeniería Química. Se puede concluir que existe una gran disparidad en cuanto a la intensidad de la enseñanza de procesos físicos entre las distintas universidades, tanto en asignaturas como en carga lectiva, aunque los contenidos generales son los indicados en la Figura 1.

La tradición de enfoque de las operaciones básicas y la distribución mencionada de la docencia parece un buen punto de partida, pero tiene el riesgo de que se pretenda impartir los principios físicos sin una adaptación al campo biotecnológico y a los estudiantes. Se precisa pues un enfoque ajustado a los estudiantes de Biotecnología y a los requerimientos específicos de este campo y a sus aplicaciones industriales. Algunos comentarios respecto a posibles deficiencias en la forma de educación podrían ser:

- A la hora de diseñar la operación unitaria, realizar simplificaciones que no son aplicables a procesos biológicos.

- Entrar en un estudio detallado de los cálculos estándar, pero no hacer mayor hincapié en los fenómenos de transporte, generales.

- Explicar operaciones que tradicionalmente se usan en la Ingeniería Química, pero que son poco utilizadas en el diseño de bioprocesos debido a las particularidades de los procesos biológicos.

- No explicar operaciones poco usadas en Ingeniería Química debido a su coste o baja capacidad, pero que sí son atractivas en Ingeniería de Bioprocesos por sus características de alta especificidad, condiciones de aplicación poco agresivas.

\section{EN BIOTECNOLOGÍA HAY QUE MOVER FLUIDOS}

La mayoría de los materiales de uso biotecnológico se manejan en fase líquida y hay que cambiarles de posición a través de operaciones como el bombeo, la agitación, mezcla o dispersión o separarlos de un sólido. Los cambios involucrados y la evolución de las velocidades se determinan a partir del Análisis del Principio de Conservación de la Cantidad de Movimiento, aunque se requiera para ello también de los Principios de Conservación de Materia y de Conservación del Momento Cinético. De ahí que frecuentemente se llame a las operaciones que se basan en aprovechar esos cambios de la velocidad como Operaciones de Cantidad de Movimiento, que será a las que nos referiremos en este apartado. El conocimiento de estas operaciones es esencial, no solamente para tratar con 


\begin{tabular}{|c|c|c|c|c|c|c|c|c|c|c|c|c|c|c|c|c|c|c|c|c|c|c|c|c|c|c|c|c|c|c|}
\hline 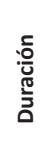 & $\frac{\bar{g}}{\frac{\pi}{4}}$ & 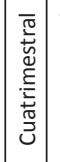 & 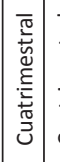 & & 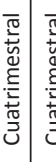 & 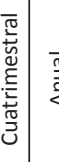 & & 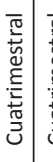 & & 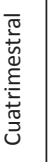 & 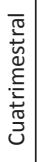 & 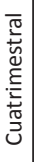 & 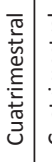 & 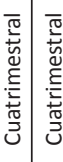 & 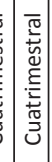 & 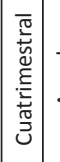 & & & 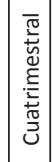 & 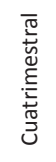 & 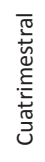 & 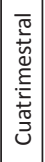 & 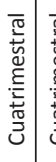 & 酸 & 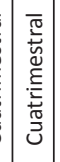 & 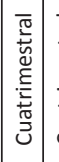 & & 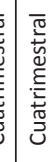 & & \\
\hline$\frac{\stackrel{0}{3}}{3}$ & $\stackrel{\circ}{\sim}$ & $\dot{m}$ & $\stackrel{\circ}{\sim}$ & $\dot{m}$ & $\mathrm{~m}$ & of & i & $\stackrel{2}{\sim}$ & Ni & $\stackrel{\circ}{\sim}$ & ì & i & $\dot{m}$ & $\stackrel{\sim}{\sim} \stackrel{\circ}{m}$ & $\stackrel{m}{m}$ & $\stackrel{\circ}{\sim}$ & $\stackrel{i}{m} \bar{z}$ & $\begin{array}{l}\stackrel{0}{z} \\
z\end{array}$ & 19 & $\stackrel{2}{\sim}$ & 임 & $\stackrel{1}{\prime}$ & $\stackrel{2}{2}$ & $\stackrel{m}{m}$ & $\stackrel{2}{\sim}$ & is & of & $s \stackrel{m}{m}$ & $\stackrel{\circ}{\sim}$ & $\stackrel{2}{\sim}$ \\
\hline يّ & $\sigma$ & 6 & 6 & & 0 & $\circ$ & $\sigma$ & & & 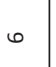 & & & n & 6 & & 0 & & $\begin{array}{lll}\frac{0}{2} & 0\end{array}$ & & 0 & 0 & 6 & & ๑ & & 6 & ๑ & \begin{tabular}{l|l}
0 & $\sigma$
\end{tabular} & $\sigma$ & \\
\hline
\end{tabular}

\begin{tabular}{|c|c|c|c|c|c|c|c|c|c|c|c|c|c|c|c|c|c|c|c|c|c|c|c|}
\hline & 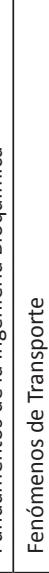 & 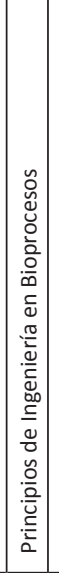 & 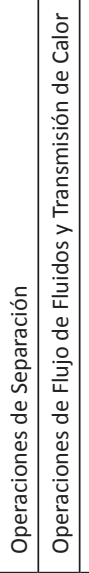 & 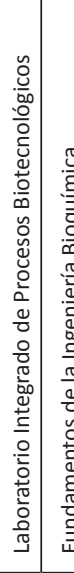 & 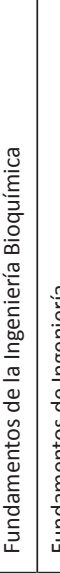 & 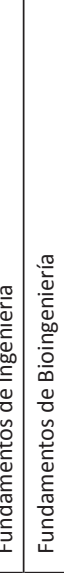 & 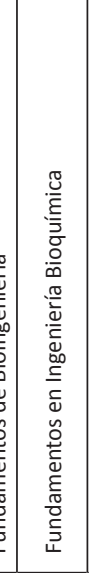 & 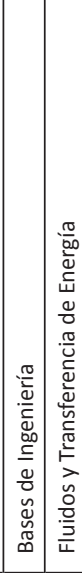 & 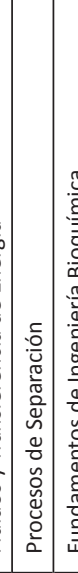 & 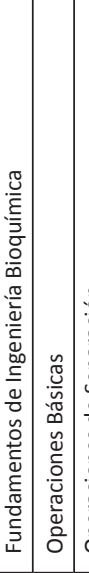 & 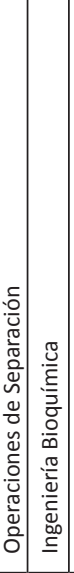 & 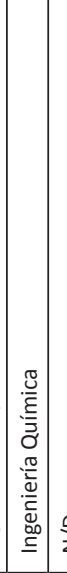 & 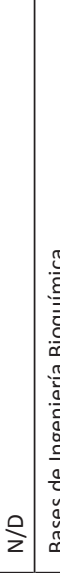 & 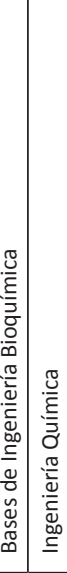 & 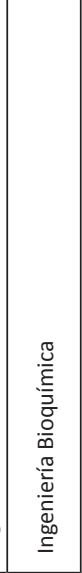 & 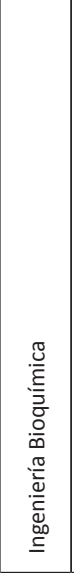 & 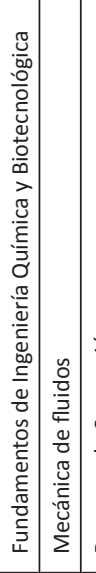 & 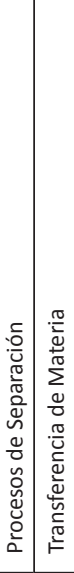 & 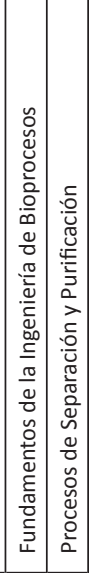 & 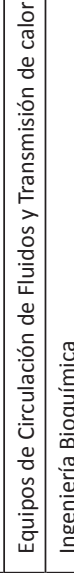 & 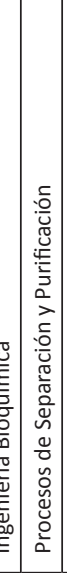 & 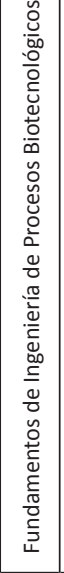 & 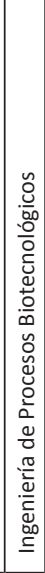 \\
\hline 8 & 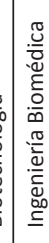 & & 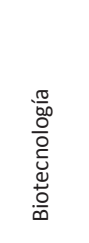 & & 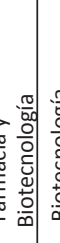 & 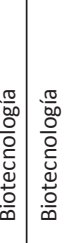 & 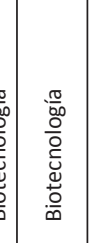 & 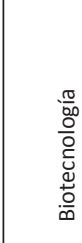 & & 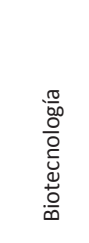 & 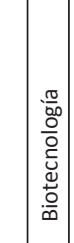 & 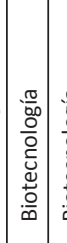 & 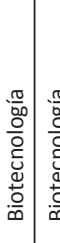 & 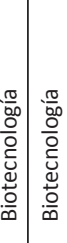 & 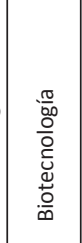 & 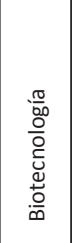 & 営 & & 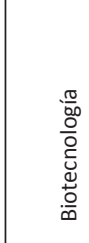 & & 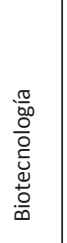 & 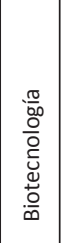 & \\
\hline & 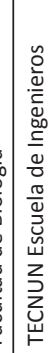 & & 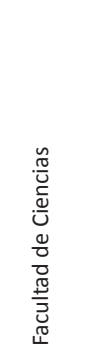 & 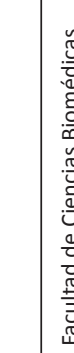 & 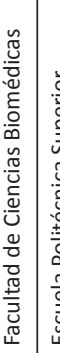 & 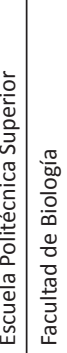 & 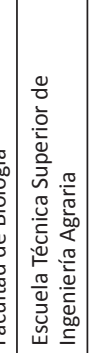 & 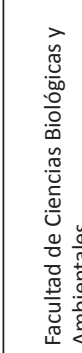 & 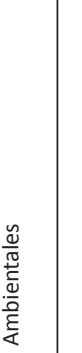 & 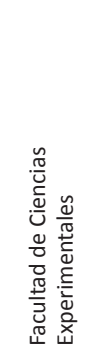 & 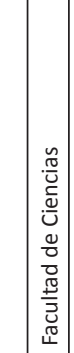 & 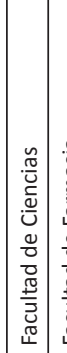 & 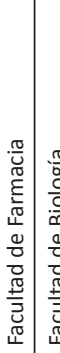 & 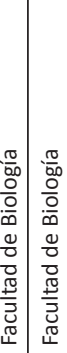 & 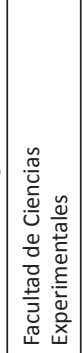 & 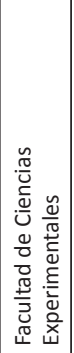 & 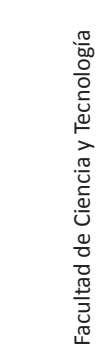 & & 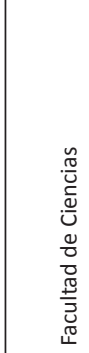 & & 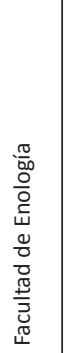 & & 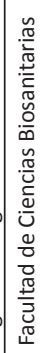 \\
\hline & 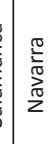 & & 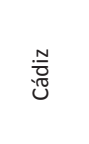 & & إ. & 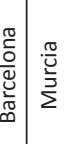 & $\frac{\frac{\pi}{0}}{\stackrel{0}{\Theta}}$ & ه్ర. & S & 意 & \begin{tabular}{|l|}
0 \\
$\frac{0}{0}$ \\
$\frac{0}{0}$ \\
\end{tabular} & 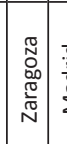 & 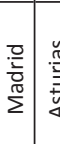 & 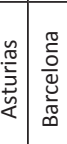 & $\begin{array}{l}\frac{\pi}{\mathrm{L}} \\
\frac{\mathrm{U}}{\mathrm{N}} \\
>\end{array}$ & 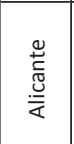 & $\sum^{N}$ & & 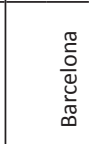 & & 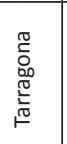 & 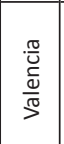 & \\
\hline & & & 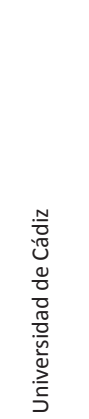 & 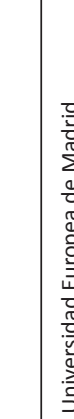 & 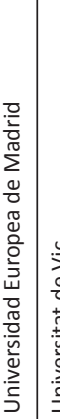 & 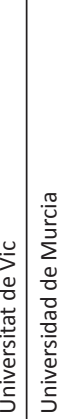 & 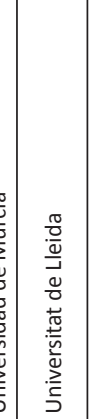 & 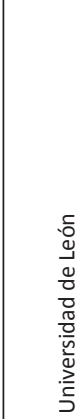 & & 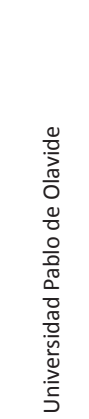 & 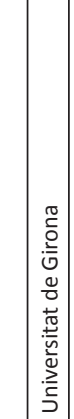 & 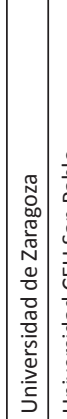 & 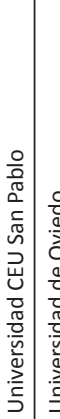 & 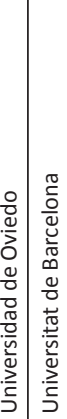 & 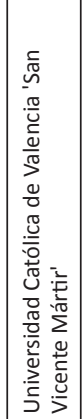 & 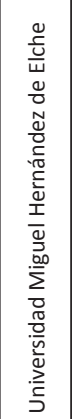 & $\frac{\frac{\pi}{0}}{\frac{0}{0}}$ & & 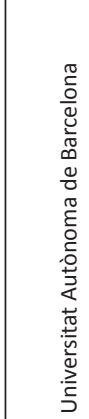 & & 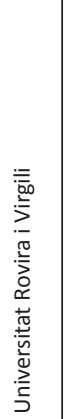 & 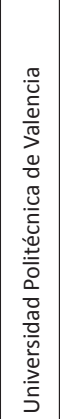 & \\
\hline
\end{tabular}


exactitud los problemas de movimiento de fluidos a través de bombas, tuberías, biorreactores..., sino también para el estudio de las operaciones unitarias que dependen de la transmisión de calor o de la transferencia de materia.

Cuando se analizan las operaciones donde hay movimiento de fluido, lo primero que debemos estudiar será el comportamiento del fluido (reología), esto es, qué esfuerzo hay que hacer en un fluido para generar cierto gradiente de velocidad o deslizamiento. Esto se consigue en los fluidos con comportamiento viscoso relacionando los esfuerzos tangenciales con el gradiente de velocidad, mientras que si el fluido tiene también un comportamiento elástico, se precisa además de la relación entre esfuerzos normales y deslizamiento.

Podemos ilustrar en este punto algunas especificidades de los procesos biológicos, muchas veces productos renovables, respecto los tradicionales (ver Figura 3):

\section{El fluido posiblemente no sea newtoniano}

El diseño de numerosas operaciones suele basarse en suponer que el fluido objeto de estudio presenta un comportamiento reológico solo viscoso de tipo newtoniano, es decir, que presenta una viscosidad constante e independiente del esfuerzo cortante. Sin embargo, los fluidos presentes en procesos biológicos tienen distintos tipos de comportamiento en función de los nutrientes, los microorganismos y el tipo de producto.
De hecho, muchos fluidos involucrados en bioprocesos precisan proporcionalmente de menor esfuerzo al aumentar el gradiente de velocidad (pseudoplásticos) o con bajo esfuerzo no se mueven (fluidos plásticos).

\section{Probablemente no tengamos un sistema monofásico}

Es cierto que esto es frecuente en procesos químicos, por ejemplo, en separaciones entre fases o en reactores heterogéneos. En procesos biológicos, la presencia de microrganismos en la fase acuosa originan un sistema sólido/líquido, al cual con frecuencia se añaden nutrientes (por ejemplo, oxígeno) o se generan productos en una fase distinta. Piénsese, si no, en que en muchos bioprocesos se parte de una materia prima sólida que se procesa en fase líquida y se expende en fase sólida.

\section{Las propiedades fisicoquímicas pueden cambiar bastante con el tiempo}

Es frecuente la elección de procesos discontinuos en el campo biotecnológico. Ello está relacionado con la obtención de productos de alto valor añadido y la gran rotación en el desarrollo de nuevos productos, lo que dificulta disponer de instalaciones continuas específicas que respondan ante la diversidad en productos y modificaciones. En procesos discontinuos cambian las composiciones con el tiempo y, en consecuencia, también las propiedades. Por ejemplo, es frecuente que cultivos celulares, inicialmente newtonianos, se conviertan primeramente en pseudoplásticos y luego en

Figura 3. Alguna de las características de los bioprocesos que van a determinar el diseño de las operaciones físicas.

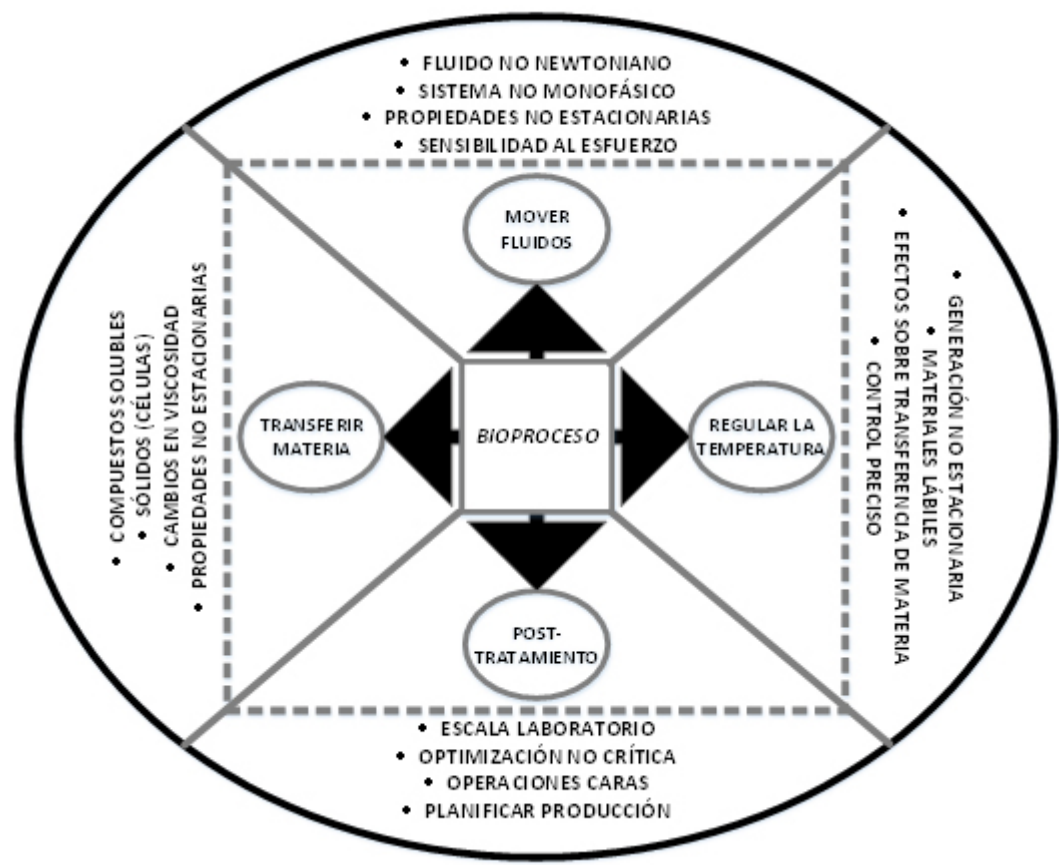


plásticos de Bingham a medida que la concentración celular aumenta. Esto conlleva la necesidad de ajustar con el tiempo las condiciones de operación con el fin de obtener los rendimientos deseables.

\section{La transformación que se busca puede ser sensible al esfuerzo cortante}

La actividad metabólica celular se puede ver reducida o incluso cesar ante esfuerzos cortantes altos. Según la teoría de Kolmogorov, si las entidades biológicas (flóculos, hifas, aglomerados...) son menores que la "distancia entre las líneas de corriente" generadas en cualquier operación unitaria de transporte de cantidad de movimiento, se deterioran menos por que van embebidas en la corriente. Pero si ambas son de magnitud análoga, la entidad se ve sometida a diferentes velocidades a uno y otro extremo, es decir, se va estirando y se deteriora. Por lo tanto, el deterioro celular va a depender de la potencia y del tamaño de la entidad biológica.

Por otra parte, el esfuerzo cortante también tiene efecto sobre las velocidades de floculación y desfloculación según esquemas como los de Argaman-Kaufman, modificando el tamaño de los flóculos y, por tanto, las capacidades de separación y de transformación de los microorganismos en su interior.

\section{LOS DISTINTOS COMPUESTOS Y BIOCATALIZADORES DEBEN AJUSTARSE A DETERMINADAS TEMPERATURAS}

Prácticamente en todas las operaciones involucradas en el diseño de un bioproceso hay unas temperaturas óptimas a las que conviene mover o transformar los materiales. Además, en muchos procesos se genera calor (en unos pocos se consume), lo que debe tenerse en cuenta a la hora de darle la temperatura adecuada al proceso durante la esterilización del medio, la conservación del bioproducto, o el control de la temperatura en el biorreactor.

Un diseño adecuado de los sistemas de intercambio de calor, un buen sistema de control de temperatura o la integración térmica pueden condicionar en gran medida la eficacia y la rentabilidad de un bioproceso. Al igual que en las operaciones de transporte de cantidad de movimiento, el diseño tradicional de operaciones unitarias basadas en la transmisión de calor para su inclusión en un bioproceso no resulta adecuado si no se consideran las especificidades de los medios biológicos (Figura 3), así:

\section{Es muy común la generación de calor cambiante con} el tiempo

Excepto en algunas reacciones enzimáticas, los procesos biológicos sonexotérmicos. El metabolismo de los microorganismos genera calor, el cual debe ser extraído del medio con el fin de mantener la temperatura adecuada. Por ello, las tasas de generación y extracción de calor en procesos discontinuos pueden variar con el tiempo en sistemas discontinuos, e incluso en algunas etapas de la operación real en procesos continuos.

Posiblemente se tenga materiales muy sensibles a cambios de temperatura

Los procesos biológicos son muy sensibles a la temperatura y su eficacia depende en gran medida de que se alcance de forma rápida y estable la temperatura óptima de operación. Temperaturas excesivas, aún por cortos tiempos, provocan la muerte celular, lo que conlleva un nuevo arranque del biorreactor. Además, temperaturas elevadas dan lugar a la desnaturalización de proteínas, lo que a su vez causa incrustaciones en las paredes del biorreactor o del cambiador.

Por último, muchos bioproductos son lábiles y se deterioran fácilmente. Esto conlleva que un gran número de operaciones unitarias basadas en la transmisión de calor y profusamente empleadas en procesos químicos, como la destilación o la evaporación, no sean aplicables, mientras que otras, como la liofilización o la congelación, ganen peso.

Probablemente se tenga que evaluar la transmisión de calor y de materia conjuntamente

Con frecuencia el diseño de las operaciones de transmisión de calor va a ser muy importante a la hora de evaluar la transferencia de materia. Por ejemplo, la solubilidad del oxígeno, clave en procesos biológicos aerobios, está fuertemente influenciada por la temperatura. Eso hace que se tengan que resolver simultáneamente ambos balances, de materia y de energía. Otra situación típica que hace necesario ambos balances es a la hora de evitar puntos de temperatura no controlada o de ausencia de nutrientes debidos a la baja conductividad térmica de los materiales biológicos.

Hay que fijarse bien en los términos importantes para el balance de energía

Muchos procesos biológicos tienen tiempos de operación largos, y en algunos periodos el calor generado puede ser pequeño. Además, la temperatura debe regularse con frecuencia en el estrecho intervalo en el que es viable un proceso biológico. Todo lo anterior conlleva la necesidad de una regulación térmica óptima. Ello hace que el calor aportado por agitación o el calor eliminado por evaporación natural o por aireación deban considerarse con frecuencia.

\section{LA TRANSFERENCIA DE MATERIA Y LOS EQUILI- BRIOS ENTRE FASES DEBEN CONOCERSE BIEN}


Cuando se consideran los procesos biológicos, las operaciones de transferencia de materia adquieren interés particular en procesos de difusión, reacciones y equilibrios en fase acuosa. Muchas operaciones están basadas en la separación de componentes de mezclas mediante transferencia desde una fase a otra usando como fuerza impulsora un gradiente de concentración. En el diseño de biorreactores, en particular en condiciones aerobias, estas operaciones se hacen particularmente importantes a la hora de asegurar un aporte continuo y estable de oxígeno al medio líquido desde un medio gaseoso (aire). Una estimación correcta de la velocidad de transferencia de oxígeno al medio a diferentes escalas y bajo unas condiciones operacionales dadas puede ser clave a la hora de deducir las rutas metabólicas seguidas por los microorganismos y de diseñar el equipo. Frecuentemente, la velocidad de transferencia de oxígeno controla la velocidad global del proceso, es decir, la capacidad del equipo a escala industrial.

Este problema ha sido muy importante en los inicios de la disciplina de Ingeniería Bioquímica o de Bioprocesos. La transferencia de oxígeno desde una burbuja de gas hasta una célula puede representarse a través de una serie de etapas y resistencias que se indican: 1) Transferencia desde el interior de la burbuja hasta la interfase gas-líquido. 2) Movimiento a través de la interfase gas-líquido. 3) Difusión a través de la capa líquida límite de la burbuja. 4) Transporte a través del medio líquido de reacción. 5) Difusión a través de la capa líquida límite de la célula. 6) Difusión a través del sólido, si la célula está en un flóculo. 7) Movimiento a través de la interfase liquido-célula. 8) Transporte a través del citoplasma hasta el lugar donde tiene lugar la reacción. 9) Reacciones bioquímicas de consumo de oxígeno y producción de $\mathrm{CO}_{2}$. 10) Transferencia de los gases producidos en dirección opuesta.

Se puede observar que las etapas 1 a 8 y 10 corresponden a procesos físicos. En general, la resistencia a la difusión a través de la capa líquida que rodea las burbujas es la etapa limitante (etapa 3). En procesos biológicos, esta resistencia depende de un gran número de factores que no suelen ser considerados durante el diseño tradicional de operaciones unitarias de transferencia de materia (Figura 3). Señalaremos también aquí algunas características específicas.

\section{Presencia de compuestos solubles en el medio cultivo}

La composición en fase líquida suele ser compleja y cambiante. En los cultivos celulares se precisan muchos nutrientes diferentes, y de composición cambiante. Esta composición afecta mucho al coeficiente volumétrico de transferencia de materia. Las sales reducen las velocidades de ascenso de las burbujas, al reducir su tamaño y disminuir los fenómenos de coalescencia, aumentando el área específica. Otros compuestos, como alcoholes y moléculas orgánicas pequeñas, también aumentan el coeficiente volumétrico de transferencia de materia, y lo mismo ocurre con muchas moléculas biológicas de alto peso molecular, especialmente aquellas con propiedades espumantes, como las proteínas.

\section{Atención al impacto de la composición en las propie- dades físicas}

La composición en fase líquida puede tener un gran impacto en propiedades como la tensión superficial. La formación de espumas, frecuentemente asociada con la presencia de proteínas, es habitual en bioprocesos, por lo que es común el empleo de agentes antiespumantes que desestabilicen la estructura de la espuma. La adición de un antiespumante al medio provoca cambios en la hidrodinámica del sistema y, como consecuencia, en las velocidades de transferencia. Estos compuestos potencian fenómenos de coalescencia, lo que provoca un aumento del diámetro medio de burbuja y de la velocidad ascensional, reduciendo el área interfacial $a$. Además, los agentes antiespumantes forman una monocapa en la interfase gas-líquido que aumenta la resistencia al paso de las moléculas de gas y reduce la movilidad de las burbujas, lo que implica una reducción del coeficiente de transferencia líquido $k_{L}$. En definitiva, la necesidad de antiespumantes puede provocar una caída drástica de las velocidades de transferencia de oxígeno.

\section{Atención a la presencia de sólidos (células) en muchas operaciones}

La presencia de componentes sólidos es inherente a los procesos fermentativos y las operaciones anexas. También pueden presentarse en sistemas con enzimas inmovilizados, o en las etapas iniciales y finales del bioproceso. El impacto, por ejemplo, de la concentración de sólidos suspendidos en la transferencia de oxígeno ha sido estudiado por numerosos autores en un amplio rango de sistemas y tipos de partículas. En todos los casos se ha observado una reducción de la velocidad de transferencia de oxígeno a medida que aumenta la concentración de sólidos. Parece probable que la acumulación de células en las proximidades de la interfase forme una capa que reduce la transferencia de oxígeno, lo que se conoce como efecto de bloqueo físico. Se cree que un aumento de la concentración de sólidos no afecta con la misma importancia a los dos parámetros del coeficiente volumétrico de transferencia, $k_{L}$ y $a$. Una mayor cantidad de sólidos aumenta los fenómenos de coalescencia de las burbujas, lo cual reduce el área interfacial $a$. Sin embargo, el efecto sobre $k_{L}$ es menos acusado. La presencia de una pe- 
queña cantidad de sólidos puede incluso mejorar en ocasiones el coeficiente de transferencia, lo cual se atribuye al efecto disruptivo de las partículas pequeñas sobre la capa límite.

\section{Cambio fuerte de propiedades al cambiar la compo- sición con el tiempo}

Si antes comentábamos el impacto de la composición de la fase acuosa en la tensión superficial, tenemos que llamar también la atención sobre su impacto en la viscosidad. Un ejemplo ilustrativo es la bioproducción de polisacáridos, como la goma xantana. Durante la fermentación, la viscosidad puede pasar desde un valor próximo a $10^{-3}$ hasta varios miles de Pa.s, lo que hace que las necesidades de bombeo, mezcla o transporte de calor o materia también vayan cambiando de forma drástica con el tiempo. Por ello, el diseño en estas condiciones requiere la adaptación con el tiempo de las condiciones de operación.

\section{Y ADAPTAR LOS PROCESOS DE TRANSPORTE AL POST-TRATAMIENTO (“DOWNSTREAM PROCESSING”)}

Las operaciones que se utilizan en el post-tratamiento suelen clasificarse en tres bloques: 1) Operaciones de separación por velocidad y en mallas; 2 ) Operaciones de separación con transferencia entre fases; 3 ) Eliminación de agua y presentación final del sólido. En el primer bloque estarían incluidas operaciones como la sedimentación/centrifugación, la flotación, la electroforesis o la diálisis, así como las operaciones con membranas. En el segundo grupo se incluirían las operaciones por retención en sólidos (como la adsorción, la cromatografía o el intercambio iónico), las de transferencia a otra fase (como la extracción o la destilación), o las separaciones por transformación de fase (como precipitación, cristalización, congelación, evaporación o liofilización, entre otras).Respecto al último bloque, debe considerarse al menos la preparación de aire y el secado, así como la mezcla de sólidos, granulación, extrusión etc. Indicaremos solo algunas características diferenciales de interés (ver Figura 3 ):

\section{Estudiar equipos de laboratorio ampliados a escala productiva}

Los bioprocesos se desarrollan con frecuencia a escala próxima a la de laboratorio; incluso para obtener producciones bastante elevadas. Ello hace que partiendo de resultados de laboratorio, al querer obtener producciones algo más elevadas, pero no excesivamente, la primera intención sea continuar con una metodología análoga, simplemente con equipos más grandes, pero no cambiando sustancialmente el método. Resultan así operaciones de laboratorio, pero en una escala mayor. Las condiciones de seguridad al trabajar en mayor escala y en producción para comercializar serán, no obstante, más exigentes, en particular con organismos de control y acreditación.

\section{La optimización de procesos quizás no sea crítica}

Suele resultar importante poner en marcha los procesos en forma rápida, y acreditarlos con organismos nacionales o internacionales. Ello hace que con frecuencia no se pueda dedicar mucho tiempo a procesos de optimización de tipo adicional que, además, pueden requerir más procesos administrativos. Por ello, a veces prima la puesta en marcha sobre la optimización exhaustiva del proceso productivo.

\section{Quizás tengamos que trabajar con operaciones que suelen considerarse caras}

La selección de las operaciones más adecuadas para cada bioproceso es una etapa fundamental en el diseño de un proceso que debe incluirse en alguna forma en la educación del biotecnólogo. Esta selección suele resultar más compleja que el dimensionado en sí de los equipos en las distintas operaciones. En esta selección debemos pensar que los bioproductos son frecuentemente de valor elevado y que, por tanto, tiene sentido considerar operaciones consideradas a priori como caras, en particular cuando se desea una pureza elevada, lo que suele ser muy frecuente en este tipo de procesos.

Tal vez haya que pensar en algunos procesos de posttratamiento discontinuos

Muchos post-tratamientos de los que se han indicado arriba suelen tratarse en los textos solo como procesos continuos, aunque en procesos biológicos para volúmenes bajos suelan llevarse a cabo en discontinuo. Puede pensarse como ejemplos en el secado o en las operaciones con membranas.

\section{Atención al planificar la producción}

La planificación de la producción es un aspecto importante. En particular, los problemas de carga y descarga de los equipos en procesos discontinuos se plantean conllevan que una correcta secuenciación de los tiempos destinados a cada tarea resulte clave a la hora de conseguir una producción fiable y ajustada a las necesidades reales.

\section{CONCLUSIONES}

La demanda de formación sobre aspectos de Diseño de Procesos Físicos u Operaciones Básicas para titulaciones relacionadas con la Biotecnología ha crecido de forma enorme. La necesidad de esta formación se 
hace evidente para los distintos estudios relacionados con el procesado de y con materiales biológicos, tales como la Biotecnología, Bioquímica, Tecnología de Alimentos, Ciencias Ambientales o Ingeniería Agronómica. Por motivos del tipo de industria biotecnológica, y también pedagógicos, el enfoque formativo debe adaptarse al tipo de titulación y alumno, y no trasplantar directamente el que se da en otras Ingenierías de Procesos. Este nuevo enfoque debe tener en cuenta conocimientos que resultan importantes para la mejora o desarrollo de bioprocesos.

El uso de materiales biológicos con unas características muy diferentes de las materias primas no renovables o tradicionales, es uno de los factores más importantes a la hora de adaptar la enseñanza. Las características de las moléculas biológicas hacen que determinadas operaciones, tradicionalmente aplicadas en otros campos(ingeniería química, mecánica, minera...) sean de difícil uso o precisen de condiciones de operación diferentes. Los medios biológicos conllevan modificaciones a la hora de diseñar sistemas de bombeo, agitación o mezcla, sobre las que hay que hacer hincapié durante la formación en particular del alumno de Biotecnología. Los procesos biológicos suelen ser también exotérmicos y muy sensibles a la temperatura de operación y a la concentración de oxígeno disuelto, por lo que un buen conocimiento de los procesos de transmisión de calor y del sistema de transferencia de materia resulta clave.

La naturaleza de los bioproductos generados, compuestos lábiles de un elevado valor añadido, es otro factor importante a tener en la adaptación del Diseño de Procesos Físicos. Operaciones de separación basadas en principios físicos poco agresivos con el producto pero con una alta especificidad, como la cromatografía o el empleo de membranas, requieren de una mayor atención.

\section{BIBLIOGRAFÍA}

Díaz Fernández, M. (2012). Ingeniería de Bioprocesos. Madrid: Paraninfo.

Gòdia Casablanca, F. y López Santín, J. (eds.). (1998). Ingeniería Bioquímica. Madrid: Síntesis. 